\section{TATRA \\ MOUNTaiNS \\ Mathematical Publications}

DOI: $10.2478 /$ tmmp-2013-0001

Tatra Mt. Math. Publ. 54 (2013), 1-18

\title{
IMPROVED STABILITY ESTIMATES FOR IMPULSIVE DELAY REACTION-DIFFUSION COHEN-GROSSBERG NEURAL NETWORKS VIA HARDY-POINCARÉ INEQUALITY
}

\author{
Haydar AkÇa - Valéry Covachev - Zlatinka Covacheva
}

\begin{abstract}
An impulsive Cohen-Grossberg neural network with time-varying and S-type distributed delays and reaction-diffusion terms is considered. By using Hardy-Poincaré inequality instead of Hardy-Sobolev inequality or just the nonpositivity of the reaction-diffusion operators, under suitable conditions in terms of $M$-matrices which involve the reaction-diffusion coefficients and the dimension and size of the spatial domain, improved stability estimates for the system with zero Dirichlet boundary conditions are obtained. Examples are given.
\end{abstract}

\section{Introduction}

Since Cohen-Grossberg neural networks [8] were proposed in 1983, extensive work has been done on this subject due to their extensive applications in the classification of patterns, associative memories, image processing, quadratic optimization, and other areas. In the implementation of neural networks, however, time delays inevitably occur due to the finite switching speed of neurons and amplifiers.

The most widely studied and used neural networks can be classified as either continuous or discrete. Recently, there has been a rather new category of neural networks which are neither purely continuous-time nor purely discrete-time. This third category of neural networks called impulsive neural networks displays a combination of characteristics of both the continuous and the discrete systems [10].

(C) 2013 Mathematical Institute, Slovak Academy of Sciences.

2010 Mathematics Subject Classification: 34A37, 35B35, 35K57, 92B20.

Keywords: Cohen-Grossberg neural networks, S-type delays, impulses, reaction-diffusion, Hardy-Poincaré inequality.

This study has been supported by the Abu Dhabi University College of Research and Graduate Studies with reference number CRGS/ICR/01-2012. 
It is well known that diffusion effect cannot be avoided in the neural networks when electrons are moving in asymmetric electromagnetic fields [16, so the activations must be considered to vary in space as well as in time. The papers [14], [15] are devoted to the exponential stability of impulsive Cohen-Grossberg neural networks with, respectively, time-varying and distributed delays and reaction-diffusion terms. In the above cited papers and many others as well as in our recent paper [4 the stability conditions were independent of the diffusion. On the other hand, in [18, [22], 23] the estimate of the exponential convergence rate depends on the reaction-diffusion.

In the present paper we consider an impulsive Cohen-Grossberg neural network with both time-varying and S-type distributed delays [5], 11, 13, 21 and reaction-diffusion terms as in [19], 22, 23] which are of a form more general than in [14, 15], and zero Dirichlet boundary conditions. By using Hardy-Poincaré inequality as in [23], under suitable conditions in terms of $M$-matrices which involve the reaction-diffusion coefficients and the dimension and size of the spatial domain, it is proved that for the system with zero Dirichlet boundary conditions the equilibrium point is globally exponentially stable. The estimate of the Lyapunov exponent is more precise than those obtained by using Hardy-Sobolev inequality or just the nonpositivity of the reaction-diffusion operators. Examples are given.

\section{Model description and preliminaries}

We consider the impulsive Cohen-Grossberg neural network with time-varying and S-type distributed delays and reaction-diffusion terms, and zero Dirichlet boundary conditions:

$$
\begin{aligned}
\frac{\partial u_{i}(t, x)}{\partial t}= & \sum_{\nu=1}^{n} \frac{\partial}{\partial x_{\nu}}\left(D_{i \nu}(t, x, u) \frac{\partial u_{i}(t, x)}{\partial x_{\nu}}\right)+\alpha_{i}\left(u_{i}(t, x)\right) \\
& \times\left[-\beta_{i}\left(u_{i}(t, x)\right) \sum_{j=1}^{m}+\sum_{j=1}^{m} a_{i j} f_{j}\left(u_{j}(t, x)\right)+\sum_{j=1}^{m} b_{i j} g_{j}\left(u_{j}\left(t-\tau_{i j}(t), x\right)\right)+\right. \\
& \left.+\sum_{j=1}^{m} c_{i j} \int_{-\infty}^{0} h_{j}\left(u_{j}(t+\theta, x)\right) d \eta_{i j}(\theta)+J_{i}\right], \quad t>0, \quad t \neq t_{k}, \quad(1) \\
\Delta u_{i}\left(t_{k}, x\right)=-B_{i k} u_{i}\left(t_{k}, x\right)+\int_{t_{k-1}-t_{k}}^{0} u_{i}\left(t_{k}+\theta\right) d \zeta_{i k}(\theta), \quad k \in \mathbb{N}, & \quad u_{i}(s, x)=\phi_{i}(s, x), \quad s \in \Omega, \quad i=\overline{1, m}, \quad(2)
\end{aligned}
$$




\section{IMPROVED ESTIMATES VIA HARDY-POINCARÉ INEQUALITY}

where $m \geq 2$ is the number of neurons in the network; $\Omega \subset \mathbb{R}^{n}$ is a bounded open set containing the origin, with smooth boundary $\partial \Omega$ and mes $\Omega>0$; $D_{i \nu}(t, x, u)>0$ are smooth functions corresponding to the transmission diffusion operator along the $i$ th neuron; $\alpha_{i}\left(u_{i}\right)$ represent amplification functions; $\beta_{i}\left(u_{i}\right)$ are appropriately behaving functions which support the stabilizing feedback term $-\alpha_{i}\left(u_{i}\right) \beta_{i}\left(u_{i}\right)$ of the $i$ th neuron; $a_{i j}, b_{i j}, c_{i j}$ denote the connection weights (or strengths) of the synaptic connections between the $j$ th neuron and the $i$ th neuron; $f_{j}\left(u_{j}\right), g_{j}\left(u_{j}\right), h_{j}\left(u_{j}\right)$ denote the activation functions of the $j$ th neuron; $J_{i}$ denotes external input to the $i$ th neuron; $\tau_{i j}(t)$ correspond to the transmission delays; the past effect of the $j$ th neuron on the $i$ th neuron is given by the Lebesgue-Stieltjes integral $\int_{-\infty}^{0} h_{j}\left(u_{j}(t+\theta, x)\right) d \eta_{i j}(\theta)$; $\Delta u_{i}\left(t_{k}, x\right)=u_{i}\left(t_{k}+0, x\right)-u_{i}\left(t_{k}-0, x\right)$ denote impulsive state displacements at fixed moments (instants) of time $t_{k}, k \in \mathbb{N}$, involving Lebesgue-Stieltjes integrals; $B_{i k}$ are real numbers. Here it is assumed that $u_{i}\left(t_{k}-0, x\right)$ and $u_{i}\left(t_{k}+0, x\right)$ denote, respectively, the left-hand and right-hand limit at $t_{k}$, and the sequence of times $\left\{t_{k}\right\}_{k=1}^{\infty}$ satisfies $0=t_{0}<t_{1}<t_{2}<\cdots<t_{k} \rightarrow+\infty$ as $k \rightarrow+\infty$. The initial data $\phi(s, x)=\left(\phi_{1}(s, x), \ldots, \phi_{m}(s, x)\right)^{T}$ are such that $\sup _{s \leq 0} \sum_{i=1}^{m} \int_{\Omega} \phi_{i}^{2}(s, x) d x<\infty$.

As usual in the theory of impulsive differential equations, at the points of discontinuity $t_{k}$ of the solution $t \mapsto u(t, x)$ we assume that $u_{i}\left(t_{k}, x\right) \equiv u_{i}\left(t_{k}-0, x\right)$ (while in 14, 15] continuity from the right is assumed). It is clear that, in general, the derivatives $\frac{\partial u_{i}}{\partial t}\left(t_{k}, x\right)$ do not exist. On the other hand, according to the first equality of (1), there do exist the limits $\frac{\partial u_{i}}{\partial t}\left(t_{k} \mp 0, x\right)$. According to the above convention, we assume $\frac{\partial u_{i}}{\partial t}\left(t_{k}, x\right) \equiv \frac{\partial u_{i}}{\partial t}\left(t_{k}-0, x\right)$.

Throughout the paper we assume that:

A1: $n \geq 3$ and the positive constants $\omega$ and $R_{\Omega}$ are such that for $x=\left(x_{1}, \ldots, x_{n}\right)^{T} \in \Omega \subset \mathbb{R}^{n}$ we have $|x|^{2}=\sum_{\nu=1}^{n} x_{\nu}^{2}<\omega^{2}$ and $\operatorname{mes}\left\{x \in \mathbb{R}^{n}:|x|<R_{\Omega}\right\}=\operatorname{mes} \Omega$.

A2: There exist constants $\underline{D}_{i}>0(i=\overline{1, m})$ such that $D_{i \nu}(t, x, u) \geq \underline{D}_{i}$ for $\nu=\overline{1, n}, t \geq 0, x \in \Omega$ and $u \in \mathbb{R}^{m}$.

A3: The amplification functions $\alpha_{i}: \mathbb{R} \rightarrow(0,+\infty)$ are continuous and bounded in the sense that $0<\underline{\alpha}_{i} \leq \alpha_{i}(u) \leq \bar{\alpha}_{i}$ for $u \in \mathbb{R}, i=\overline{1, m}$.

A4: The stabilizing functions $\beta_{i}: \mathbb{R} \rightarrow \mathbb{R}$ are continuous and monotone increasing, namely, $0<\underline{\beta}_{i} \leq \frac{\beta_{i}(u)-\beta_{i}(v)}{u-v}$ for $u, v \in \mathbb{R}, u \neq v, i=\overline{1, m}$.

A5: For the activation functions $f_{i}(u), g_{i}(u), h_{i}(u)$ there exist positive constants $F_{i}, G_{i}, H_{i}$ such that

$$
F_{i}=\sup _{u \neq v}\left|\frac{f_{i}(u)-f_{i}(v)}{u-v}\right|, \quad G_{i}=\sup _{u \neq v}\left|\frac{g_{i}(u)-g_{i}(v)}{u-v}\right|, \quad H_{i}=\sup _{u \neq v}\left|\frac{h_{i}(u)-h_{i}(v)}{u-v}\right|
$$

for all $u, v \in \mathbb{R}, u \neq v, i=\overline{1, m}$. 
A6: $\tau_{i j}(t)$ satisfy $0 \leq \tau_{i j}(t) \leq \tau_{i j}, 0 \leq \dot{\tau}_{i j}(t) \leq \mu_{i j}<1(i, j=\overline{1, m})$.

A7: $\eta_{i j}(\theta)(i, j=\overline{1, m}), \zeta_{i k}(\theta)(i=\overline{1, m}, k \in \mathbb{N})$ are nondecreasing bounded variation functions on $(-\infty, 0]$ and $\left[t_{k-1}-t_{k}, 0\right]$, respectively, and

$$
\int_{-\infty}^{0} e^{-\lambda \theta} d \eta_{i j}(\theta)=K_{i j}(\lambda)
$$

are continuous functions on $\left[0, \lambda_{0}\right)$ for some $\lambda_{0}>0$ and $K_{i j}(0)=1$ (without loss of generality).

Due to the zero Dirichlet boundary conditions the system (1) can have just one equilibrium point $\mathbf{0}=(0,0, \ldots, 0)^{T}$. It is really an equilibrium point of the system (11) if and only if

$$
-\beta_{i}(0)+\sum_{j=1}^{m}\left(a_{i j} f_{j}(0)+b_{i j} g_{j}(0)+c_{i j} h_{j}(0)\right)+J_{i}=0, \quad i=\overline{1, m} .
$$

From equations (11) and (3) we deduce

$$
\begin{aligned}
\frac{\partial u_{i}(t, x)}{\partial t}= & \sum_{\nu=1}^{n} \frac{\partial}{\partial x_{\nu}}\left(D_{i \nu}(t, x, u) \frac{\partial u_{i}(t, x)}{\partial x_{\nu}}\right)+\alpha_{i}\left(u_{i}(t, x)\right) \\
& \times\left[-\tilde{\beta}_{i}\left(u_{i}(t, x)\right) \sum_{j=1}^{m}+\sum_{j=1}^{m} a_{i j} \tilde{f}_{j}\left(u_{j}(t, x)\right)+\sum_{j=1}^{m} b_{i j} \tilde{g}_{j}\left(u_{j}\left(t-\tau_{i j}(t), x\right)\right)+\right. \\
& \left.+\sum_{j=1}^{m} c_{i j} \int_{-\infty}^{0} \tilde{h}_{j}\left(u_{j}(t+\theta, x)\right) d \eta_{i j}(\theta)\right], \quad t>0, \quad t \neq t_{k},
\end{aligned}
$$

where

$$
\begin{gathered}
\tilde{\beta}_{i}(u)=\beta_{i}(u)-\beta_{i}(0), \quad \tilde{f}_{i}(u)=f_{i}(u)-f_{i}(0), \quad \tilde{g}_{i}(u)=g_{i}(u)-g_{i}(0), \\
\tilde{h}_{i}(u)=h_{i}(u)-h_{i}(0), \quad i=\overline{1, m} .
\end{gathered}
$$

Now conditions A4, A5 imply

$$
\tilde{\beta}_{i}(u) u \geq \underline{\beta}_{i} u^{2}, \quad\left|\tilde{f}_{i}(u)\right| \leq F_{i}|u|, \quad\left|\tilde{g}_{i}(u)\right| \leq G_{i}|u|, \quad\left|\tilde{h}_{i}(u)\right| \leq H_{i}|u|
$$

for all $u \in \mathbb{R}$ and $i=\overline{1, m}$.

Denote

$$
\left\|u_{i}(t, \cdot)\right\|=\left(\int_{\Omega} u_{i}^{2}(t, x) d x\right)^{1 / 2} .
$$




\section{IMPROVED ESTIMATES VIA HARDY-POINCARÉ INEQUALITY}

Definition 1. The equilibrium point $u=\mathbf{0}$ of the system (10), (2) is said to be globally exponentially stable (with Lyapunov exponent $\lambda$ ) if there exist constants $\lambda>0$ and $M \geq 1$ such that for any solution $u(t, x)=\left(u_{1}(t, x), \ldots, u_{m}(t, x)\right)^{T}$ of the system (11), (2) we have

$$
\sum_{i=1}^{m}\left\|u_{i}(t, \cdot)\right\| \leq M \sup _{s \leq 0} \sum_{i=1}^{m}\left\|\phi_{i}(s, \cdot)\right\| e^{-\lambda t} \quad \text { for all } \quad t \geq 0, \quad x \in \Omega .
$$

Definition $2(\underline{6}])$. A real matrix $A=\left(a_{i j}\right)_{m \times m}$ is said to be an $M$-matrix if $a_{i j} \leq 0$ for $i, j=\overline{1, m}, i \neq j$ and all successive principal minors of $A$ are positive.

Lemma 1 ([6]). Let $A=\left(a_{i j}\right)_{m \times m}$ be a real matrix with nonpositive off-diagonal elements. Then $A$ is an $M$-matrix if and only if one of the following conditions holds:

(1) There exists a vector $\xi=\left(\xi_{1}, \xi_{2}, \ldots, \xi_{m}\right)^{T}$ with $\xi_{i}>0$ such that every component of $\xi^{T} A$ is positive, i.e., $\sum_{i=1}^{m} \xi_{i} a_{i j}>0, j=\overline{1, m}$.

(2) There exists a vector $\xi=\left(\xi_{1}, \xi_{2}, \ldots, \xi_{m}\right)^{T}$ with $\xi_{i}>0$ such that every component of $A \xi$ is positive, i.e., $\sum_{j=1}^{m} a_{i j} \xi_{j}>0, i=\overline{1, m}$.

For more details about $M$-matrices the reader is referred to [9], [12].

Further on we will need the following lemma.

Lemma 2 (Hardy-Poincaré inequality [7]). Let $\Omega \subset \mathbb{R}^{n}(n \geq 3)$ be a bounded open set containing the origin and $u \in H_{0}^{1}(\Omega)$. Then

$$
\int_{\Omega}|\nabla u|^{2} d x \geq\left(\frac{n-2}{2}\right)^{2} \int_{\Omega} \frac{u^{2}}{|x|^{2}} d x+\frac{\Lambda_{2}}{R_{\Omega}^{2}} \int_{\Omega} u^{2} d x,
$$

where $\Lambda_{2}=5.783 \ldots$ is the first eigenvalue of the Dirichlet Laplacian of the unit disc in $\mathbb{R}^{2}$ and $R_{\Omega}$ is the radius of a ball in $\mathbb{R}^{n}$ having the same measure as $\Omega$.

Hardy-Poincaré inequality implies Hardy-Sobolev inequality [1]

$$
\int_{\Omega}|\nabla u|^{2} d x \geq\left(\frac{n-2}{2}\right)^{2} \int_{\Omega} \frac{u^{2}}{|x|^{2}} d x .
$$

Now let us introduce the following matrices: $\underline{D}=\operatorname{diag}\left(\underline{D}_{1}, \ldots, \underline{D}_{m}\right)$,

$$
\begin{gathered}
\underline{\alpha}=\operatorname{diag}\left(\underline{\alpha}_{1}, \ldots, \underline{\alpha}_{m}\right), \quad \bar{\alpha}=\operatorname{diag}\left(\bar{\alpha}_{1}, \ldots, \bar{\alpha}_{m}\right), \quad \underline{\beta}=\operatorname{diag}\left(\underline{\beta}_{1}, \ldots, \underline{\beta}_{m}\right), \\
F=\operatorname{diag}\left(F_{1}, \ldots, F_{m}\right), \quad G=\operatorname{diag}\left(G_{1}, \ldots, G_{m}\right), \quad H=\operatorname{diag}\left(H_{1}, \ldots, H_{m}\right), \\
|A|=\left(\left|a_{i j}\right|\right)_{m \times m}, \quad|B(\mu)|=\left(\frac{\left|b_{i j}\right|}{1-\mu_{i j}}\right)_{m \times m}, \quad|C|=\left(\left|c_{i j}\right|\right)_{m \times m} .
\end{gathered}
$$




\section{Main results}

TheOREM 1. Suppose that the system (1), (2) satisfies assumptions A1-A7 and equalities (3) hold. If there exists a vector $\xi=\left(\xi_{1}, \ldots, \xi_{m}\right)^{T}$ with $\xi_{i}>0$ and a number $\lambda \in\left(0, \lambda_{0}\right)$ such that

$$
\begin{aligned}
& \sum_{i=1}^{m}\left\{\left[\lambda-\left(\frac{(n-2)^{2}}{4 \omega^{2}}+\frac{\Lambda_{2}}{R_{\Omega}^{2}}\right) \underline{D}_{i}-\underline{\alpha}_{i} \underline{\beta}_{i}\right] \delta_{i j}+\right. \\
& \left.\quad+\bar{\alpha}_{i}\left[\left|a_{i j}\right| F_{j}+\left|b_{i j}\right| G_{j} \frac{e^{\lambda \tau_{i j}}}{1-\mu_{i j}}+\left|c_{i j}\right| H_{j} K_{i j}(\lambda)\right]\right\} \xi_{i}<0, \quad j=\overline{1, m}
\end{aligned}
$$

where

$$
\delta_{i i}=1, \quad \delta_{i j}=0 \quad \text { for } \quad j \neq i,
$$

then there exists a constant $M \geq 1$ such that for any solution $u(t, x)=\left(u_{1}(t, x), \ldots\right.$ $\left.\ldots, u_{m}(t, x)\right)^{T}$ of the system (11), (2) we have

$$
\begin{aligned}
\sum_{i=1}^{m}\left\|u_{i}(t, \cdot)\right\| & \\
\leq M e^{-\lambda t} \prod_{k=1}^{i(0, t)}\left(\max _{i=1, m}\left|1-B_{i k}\right|+\max _{i=1, m} \int_{t_{k-1}-t_{k}}^{0} e^{-\lambda \theta} d \zeta_{i k}(\theta)\right) & \\
& \quad \sup _{s \leq 0} \sum_{i=1}^{m}\left\|u_{i}(s, \cdot)\right\|, \quad t \geq 0,
\end{aligned}
$$

where $i(0, t)=\max \left\{k \in\{0\} \cup \mathbb{N}: t_{k}<t\right\}$ is the number of instants of impulse effect $t_{k}$ in the interval $(0, t)$.

Remark 1. If in the subsequent proof we choose to use Hardy-Sobolev inequality rather than Hardy-Poincaré inequality, the inequalities (5) should be replaced by

$$
\begin{aligned}
& \sum_{i=1}^{m}\left\{\left[\lambda-\left(\frac{n-2}{2 \omega}\right)^{2} \underline{D}_{i}-\underline{\alpha}_{i} \underline{\beta}_{i}\right] \delta_{i j}+\right. \\
& \left.\quad+\bar{\alpha}_{i}\left[\left|a_{i j}\right| F_{j}+\left|b_{i j}\right| G_{j} \frac{e^{\lambda \tau_{i j}}}{1-\mu_{i j}}+\left|c_{i j}\right| H_{j} K_{i j}(\lambda)\right]\right\} \xi_{i}<0, \quad j=\overline{1, m} .
\end{aligned}
$$

Further on, if we use just the nonpositivity of the reaction-diffusion operators, 
then inequalities (5) should be replaced by

$$
\begin{aligned}
& \sum_{i=1}^{m}\left\{\left(\lambda-\underline{\alpha}_{i} \underline{\beta}_{i}\right) \delta_{i j}+\bar{\alpha}_{i}\left[\left|a_{i j}\right| F_{j}+\right.\right.\left|b_{i j}\right| G_{j} \frac{e^{\lambda \tau_{i j}}}{1-\mu_{i j}}+ \\
&\left.\left.+\left|c_{i j}\right| H_{j} K_{i j}(\lambda)\right]\right\} \xi_{i}<0 \quad \text { for } j=\overline{1, m}
\end{aligned}
$$

It is clear that if $\xi$ and $\lambda$ satisfy inequalities (7) or (8), they satisfy (5). On the other hand, we can find $\xi$ and $\lambda$ satisfying inequalities (5) but not satisfying any of the sets of inequalities (17) and (8). Thus by using Hardy-Poincaré inequality, we can prove global exponential stability with a larger Lyapunov exponent than by using Hardy-Sobolev inequality or the nonpositivity of the reaction-diffusion operators, and in some cases when the last two methods do not work we can still prove global exponential stability.

Proof. Let us note that there exists a vector $\xi=\left(\xi_{1}, \ldots, \xi_{m}\right)^{T}$ with $\xi_{i}>0$ and a number $\lambda \in\left(0, \lambda_{0}\right)$ such that inequalities (5) hold if and only if

$$
\mathcal{A}=\left(\frac{(n-2)^{2}}{4 \omega^{2}}+\frac{\Lambda_{2}}{R_{\Omega}^{2}}\right) \underline{D}+\underline{\alpha} \underline{\beta}-\bar{\alpha}(|A| F+|B(\mu)| G+|C| H)
$$

is an $M$-matrix. In fact, if $\mathcal{A}$ is an $M$-matrix, from Lemma 1 there exists a vector $\xi>0$ such that every component of $-\xi^{T} \mathcal{A}$ is negative. By continuity, there exists a $\lambda \in\left(0, \lambda_{0}\right)$ such that inequalities (5) hold. Conversely, if (5) hold for some $\lambda^{*} \in\left(0, \lambda_{0}\right)$, then they still hold for all $\lambda \in\left[0, \lambda^{*}\right]$. For $\lambda=0$, from Lemma 1$]$ we deduce that $\mathcal{A}$ is an $M$-matrix.

First we shall derive the estimate

$$
\begin{aligned}
D^{+}\left\|u_{i}(t, \cdot)\right\| \leq & -\left[\left(\frac{(n-2)^{2}}{4 \omega^{2}}+\frac{\Lambda_{2}}{R_{\Omega}^{2}}\right) \underline{D}_{i}+\underline{\alpha}_{i} \underline{\beta}_{i}\right]\left\|u_{i}(t, \cdot)\right\| \\
& +\bar{\alpha}_{i} \sum_{j=1}^{m}\left\{\left|a_{i j}\right| F_{j}\left\|u_{j}(t, \cdot)\right\|+\left|b_{i j}\right| G_{j}\left\|u_{j}\left(t-\tau_{i j}(t), \cdot\right)\right\|+\right. \\
& \left.+\left|c_{i j}\right| H_{j} \int_{-\infty}^{0}\left\|u_{j}(t+\theta, \cdot)\right\| d \eta_{i j}(\theta)\right\}, \quad t>0, \quad t \neq t_{k},
\end{aligned}
$$

where $D^{+}$denotes the upper Dini derivative. 


\section{HAYDAR AKÇA — VALÉRY COVACHEV — ZLATINKA COVACHEVA}

Let $t$ be such that $\left\|u_{i}(t, \cdot)\right\| \neq 0$. We multiply the $i$ th differential equation in (4) by $u_{i}(t, x)$ and integrate over the domain $\Omega$

$$
\begin{aligned}
\frac{1}{2} \frac{d}{d t} \int_{\Omega} u_{i}^{2}(t, x) d x= & \int_{\Omega} \sum_{\nu=1}^{n} \frac{\partial}{\partial x_{\nu}}\left(D_{i \nu}(t, x, u) \frac{\partial u_{i}(t, x)}{\partial x_{\nu}}\right) u_{i}(t, x) d x \\
& -\int_{\Omega} \alpha_{i}\left(u_{i}(t, x)\right) \tilde{\beta}_{i}\left(u_{i}(t, x)\right) u_{i}(t, x) d x \\
& +\int_{\Omega} \alpha_{i}\left(u_{i}(t, x)\right) u_{i}(t, x) \sum_{j=1}^{m} a_{i j} \tilde{f}_{j}\left(u_{j}(t, x)\right) d x \\
& +\int_{\Omega} \alpha_{i}\left(u_{i}(t, x)\right) u_{i}(t, x) \sum_{j=1}^{m} b_{i j} \tilde{g}_{j}\left(u_{j}\left(t-\tau_{i j}(t), x\right)\right) d x \\
& +\int_{\Omega} \alpha_{i}\left(u_{i}(t, x)\right) u_{i}(t, x) \sum_{j=1}^{m} c_{i j} \int_{-\infty}^{0} \tilde{h}_{j}\left(u_{j}(t+\theta, x)\right) d \eta_{i j}(\theta) d x
\end{aligned}
$$

By using Green's formula, the zero Dirichlet boundary conditions, Lemma 2 and the assumptions A1, A2 we have

$$
\begin{aligned}
& \int_{\Omega} \sum_{\nu=1}^{n} \frac{\partial}{\partial x_{\nu}}\left(D_{i \nu}(t, x, u) \frac{\partial u_{i}(t, x)}{\partial x_{\nu}}\right) u_{i}(t, x) d x \\
& =-\int_{\Omega} \sum_{\nu=1}^{n} D_{i \nu}(t, x, u)\left(\frac{\partial u_{i}(t, x)}{\partial x_{\nu}}\right)^{2} d x \leq-\underline{D}_{i} \int_{\Omega} \sum_{\nu=1}^{n}\left(\frac{\partial u_{i}(t, x)}{\partial x_{\nu}}\right)^{2} d x \\
& =-\underline{D}_{i} \int_{\Omega}\left|\nabla u_{i}(t, x)\right|^{2} d x \leq-\underline{D}_{i}\left[\left(\frac{n-2}{2}\right)^{2} \int_{\Omega} \frac{u_{i}^{2}(t, x)}{|x|^{2}} d x+\frac{\Lambda_{2}}{R_{\Omega}^{2}} \int_{\Omega} u_{i}^{2}(t, x) d x\right] \\
& \leq-\left(\frac{(n-2)^{2}}{4 \omega^{2}}+\frac{\Lambda_{2}}{R_{\Omega}^{2}}\right) \underline{D}_{i} \int_{\Omega} u_{i}^{2}(t, x) d x=-\left(\frac{(n-2)^{2}}{4 \omega^{2}}+\frac{\Lambda_{2}}{R_{\Omega}^{2}}\right) \underline{D}_{i}\left\|u_{i}(t, \cdot)\right\|^{2} .
\end{aligned}
$$

If we prefer to use Hardy-Sobolev inequality, we obtain

$$
\int_{\Omega} \sum_{\nu=1}^{n} \frac{\partial}{\partial x_{\nu}}\left(D_{i \nu}(t, x, u) \frac{\partial u_{i}(t, x)}{\partial x_{\nu}}\right) u_{i}(t, x) d x \leq-\left(\frac{n-2}{2 \omega}\right)^{2} \underline{D}_{i}\left\|u_{i}(t, \cdot)\right\|^{2}
$$

and we can complete the proof by using inequalities (7). 


\section{IMPROVED ESTIMATES VIA HARDY-POINCARÉ INEQUALITY}

Finally, in the case of zero Dirichlet or Neumann boundary conditions and no restrictions on the dimension of the spatial domain $\Omega$, we have

$$
\begin{aligned}
\int_{\Omega} \sum_{\nu=1}^{n} \frac{\partial}{\partial x_{\nu}}\left(D_{i \nu}(t, x, u) \frac{\partial u_{i}(t, x)}{\partial x_{\nu}}\right) & u_{i}(t, x) d x \\
= & -\int_{\Omega} \sum_{\nu=1}^{n} D_{i \nu}(t, x, u)\left(\frac{\partial u_{i}(t, x)}{\partial x_{\nu}}\right)^{2} d x \leq 0
\end{aligned}
$$

and we can complete the proof by using inequalities (8) .

Next we have

$$
\begin{gathered}
\int_{\Omega} \alpha_{i}\left(u_{i}(t, x)\right) \tilde{\beta}_{i}\left(u_{i}(t, x)\right) u_{i}(t, x) d x \geq \underline{\alpha}_{i} \underline{\beta}_{i} \int_{\Omega} u_{i}^{2}(t, x) d x=\underline{\alpha}_{i} \underline{\beta_{i}}\left\|u_{i}(t, \cdot)\right\|^{2} ; \\
\int_{\Omega} \alpha_{i}\left(u_{i}(t, x)\right) u_{i}(t, x) \sum_{j=1}^{m} a_{i j} \tilde{f}_{j}\left(u_{j}(t, x)\right) d x \\
\leq \bar{\alpha}_{i} \sum_{j=1}^{m}\left|a_{i j}\right| \int_{\Omega}\left|u_{i}(t, x)\right| F_{j}\left|u_{j}(t, x)\right| d x \\
\leq \bar{\alpha}_{i} \sum_{j=1}^{m}\left|a_{i j}\right| F_{j}\left(\int_{\Omega} u_{i}^{2}(t, x) d x\right)^{1 / 2}\left(\int_{\Omega} u_{j}^{2}(t, x) d x\right)^{1 / 2} \\
=\bar{\alpha}_{i} \sum_{j=1}^{m}\left|a_{i j}\right| F_{j}\left\|u_{i}(t, \cdot)\right\|\left\|u_{j}(t, \cdot)\right\| .
\end{gathered}
$$

Similarly,

$$
\begin{aligned}
& \int_{\Omega} \alpha_{i}\left(u_{i}(t, x)\right) u_{i}(t, x) \sum_{j=1}^{m} b_{i j} \tilde{g}_{j}\left(u_{j}\left(t-\tau_{i j}(t), x\right)\right) d x \\
& \leq \bar{\alpha}_{i} \sum_{j=1}^{m}\left|b_{i j}\right| G_{j}\left\|u_{i}(t, \cdot)\right\|\left\|u_{j}\left(t-\tau_{i j}(t), \cdot\right)\right\|
\end{aligned}
$$

and

$$
\begin{aligned}
& \int_{\Omega} \alpha_{i}\left(u_{i}(t, x)\right) u_{i}(t, x) \sum_{j=1}^{m} c_{i j} \int_{-\infty}^{0} \tilde{h}_{j}\left(u_{j}(t+\theta, x)\right) d \eta_{i j}(\theta) d x \\
& \leq \bar{\alpha}_{i} \sum_{j=1}^{m}\left|c_{i j}\right| H_{j}\left\|u_{i}(t, \cdot)\right\| \int_{-\infty}^{0}\left\|u_{j}(t+\theta, \cdot)\right\| d \eta_{i j}(\theta) .
\end{aligned}
$$


Combining the above inequalities, we obtain

$$
\begin{aligned}
\frac{1}{2} \frac{d}{d t}\left\|u_{i}(t, \cdot)\right\|^{2} \leq & -\left[\left(\frac{(n-2)^{2}}{4 \omega^{2}}+\frac{\Lambda_{2}}{R_{\Omega}^{2}}\right) \underline{D}_{i}+\underline{\alpha}_{i} \underline{\beta}_{i}\right]\left\|u_{i}(t, \cdot)\right\|^{2} \\
& +\bar{\alpha}_{i} \sum_{j=1}^{m}\left\{\left|a_{i j}\right| F_{j}\left\|u_{j}(t, \cdot)\right\|+\left|b_{i j}\right| G_{j}\left\|u_{j}\left(t-\tau_{i j}(t), \cdot\right)\right\|+\right. \\
& \left.+\left|c_{i j}\right| H_{j} \int_{-\infty}^{0}\left\|u_{j}(t+\theta, \cdot)\right\| d \eta_{i j}(\theta)\right\}\left\|u_{i}(t, \cdot)\right\|
\end{aligned}
$$

which implies (9) in view of $\left\|u_{i}(t, \cdot)\right\| \neq 0$.

Next, let us suppose that $t$ is such that $\left\|u_{i}(t, \cdot)\right\|=0$. If this equality holds for all $t$ in some open interval, then $\frac{d}{d t}\left\|u_{i}(t, \cdot)\right\|=0$ in this interval, and inequality (9) reduces to

$$
\begin{aligned}
0 \leq \bar{\alpha}_{i} \sum_{j=1}^{m}\left\{\left|a_{i j}\right| F_{j}\left\|u_{j}(t, \cdot)\right\|\right. & +\left|b_{i j}\right| G_{j}\left\|u_{j}\left(t-\tau_{i j}(t), \cdot\right)\right\|+ \\
& \left.+\left|c_{i j}\right| H_{j} \int_{-\infty}^{0}\left\|u_{j}(t+\theta, \cdot)\right\| d \eta_{i j}(\theta)\right\},
\end{aligned}
$$

which is trivially satisfied. If this is not the case, we can find a sequence of times $\left\{t_{l}^{\prime}\right\}$ such that

$$
t_{l}^{\prime} \rightarrow t \quad \text { and } \quad\left\|u_{i}\left(t_{l}^{\prime}, \cdot\right)\right\| \neq 0
$$

Then the validity of inequality (9) for $t_{l}^{\prime}, l \in \mathbb{N}$ implies its validity for $t$.

If we introduce the notation

$$
y_{i}(t)=e^{\lambda t}\left\|u_{i}(t, \cdot)\right\|,
$$

then from inequality (9) which we just proved we find

$$
\begin{aligned}
D^{+} y_{i}(t) \leq & {\left[\lambda-\left(\frac{(n-2)^{2}}{4 \omega^{2}}+\frac{\Lambda_{2}}{R_{\Omega}^{2}}\right) \underline{D}_{i}-\underline{\alpha}_{i} \underline{\beta}_{i}\right] y_{i}(t) } \\
& +\bar{\alpha}_{i} \sum_{j=1}^{m}\left\{\left|a_{i j}\right| F_{j} y_{j}(t)+\left|b_{i j}\right| G_{j} y_{j}\left(t-\tau_{i j}(t)\right) e^{\lambda \tau_{i j}(t)}+\right. \\
& \left.+\left|c_{i j}\right| H_{j} \int_{-\infty}^{0} e^{-\lambda \theta} y_{j}(t+\theta) d \eta_{i j}(\theta)\right\} .
\end{aligned}
$$


We consider a Lyapunov functional

$$
\begin{aligned}
V(t)=\sum_{i=1}^{m}\left\{y_{i}(t)+\bar{\alpha}_{i} \sum_{j=1}^{m}\left|b_{i j}\right| G_{j} \frac{e^{\lambda \tau_{i j}}}{1-\mu_{i j}} \int_{t-\tau_{i j}(t)}^{t} y_{j}(s) d s+\right. \\
\left.+\bar{\alpha}_{i} \sum_{j=1}^{m}\left|c_{i j}\right| H_{j} \int_{-\infty}^{0} e^{-\lambda \theta}\left(\int_{t+\theta}^{t} y_{j}(s) d s\right) d \eta_{i j}(\theta)\right\} \xi_{i}
\end{aligned}
$$

where $\lambda$ and $\xi_{i}, i=\overline{1, m}$, are as in inequalities (5).

We note that $V(t) \geq 0$ for $t \geq 0$ and

$$
V(0) \leq M \sum_{i=1}^{m} \sup _{s \leq 0} y_{i}(s)
$$

with

$$
\begin{aligned}
M=\max _{i=\overline{1, m}}\left\{\xi_{i}+G_{i} \sum_{j=1}^{m}\left|b_{j i}\right| \frac{\bar{\alpha}_{j} e^{\lambda \tau_{j i}} \tau_{j i}}{1-\mu_{j i}} \xi_{j}+\right. & \\
& \left.+H_{i} \sum_{j=1}^{m}\left|c_{j i}\right| \bar{\alpha}_{j} \int_{-\infty}^{0} e^{-\lambda \theta}(-\theta) d \eta_{j i}(\theta) \xi_{j}\right\} .
\end{aligned}
$$

The above integral is convergent because of $\lambda<\lambda_{0}$.

Calculating the rate of change of $V(t)$ along the solutions of system (10), by using successively the inequalities (10), (5) and the condition A6 we obtain

$$
\begin{aligned}
D^{+} V(t) \leq \sum_{j=1}^{m} y_{j}(t) \sum_{i=1}^{m}\{ & {\left[\lambda-\left(\frac{(n-2)^{2}}{4 \omega^{2}}+\frac{\Lambda_{2}}{R_{\Omega}^{2}}\right) \underline{D}_{i}-\underline{\alpha}_{i} \underline{\beta}_{i}\right] \delta_{i j}+} \\
+ & \left.\bar{\alpha}_{i}\left[\left|a_{i j}\right| F_{j}+\left|b_{i j}\right| G_{j} \frac{e^{\lambda \tau_{i j}}}{1-\mu_{i j}}+\left|c_{i j}\right| H_{j} K_{i j}(\lambda)\right]\right\} \xi_{i} \\
& +\sum_{i=1}^{m} \bar{\alpha}_{i} \xi_{i} \sum_{j=1}^{m}\left|b_{i j}\right| G_{j} y_{j}\left(t-\tau_{i j}(t)\right)\left(e^{\lambda \tau_{i j}(t)}-e^{\lambda \tau_{i j}} \frac{1-\dot{\tau}_{i j}(t)}{1-\mu_{i j}}\right) \leq 0 .
\end{aligned}
$$

This implies that $V(t)$ is nonincreasing on every interval $\left(t_{k-1}, t_{k}\right], k \in \mathbb{N}$, thus

$$
V(t) \leq V\left(t_{k-1}+0\right) \quad \text { for } \quad t_{k-1}<t \leq t_{k} .
$$

In particular,

$$
V\left(t_{k}\right) \leq V\left(t_{k-1}+0\right), \quad k \in \mathbb{N}
$$


Further on, for $k \in \mathbb{N}$ we find, successively,

$$
\begin{gathered}
u_{i}\left(t_{k}+0, x\right)=\left(1-B_{i k}\right) u_{i}\left(t_{k}, x\right)+\int_{t_{k-1}-t_{k}}^{0} u_{i}\left(t_{k}+\theta, x\right) d \zeta_{i k}(\theta), \\
\left\|u_{i}\left(t_{k}+0, \cdot\right)\right\| \leq\left|1-B_{i k}\right|\left\|u_{i}\left(t_{k}, \cdot\right)\right\|+\int_{t_{k-1}-t_{k}}^{0}\left\|u_{i}\left(t_{k}+\theta, \cdot\right)\right\| d \zeta_{i k}(\theta)
\end{gathered}
$$

and

$$
y_{i}\left(t_{k}+0\right) \leq\left|1-B_{i k}\right| y_{i}\left(t_{k}\right)+\int_{t_{k-1}-t_{k}}^{0} e^{-\lambda \theta} y_{i}\left(t_{k}+\theta\right) d \zeta_{i k}(\theta) .
$$

Making use of (12) and (13), we obtain

$$
\begin{aligned}
V\left(t_{k}+0\right) & \leq \max _{i=\overline{1, m}}\left|1-B_{i k}\right| V\left(t_{k}\right)+\max _{i=\overline{1, m}} \int_{t_{k-1}-t_{k}}^{0} e^{-\lambda \theta} d \zeta_{i k}(\theta) V\left(t_{k-1}+0\right) \\
& \leq\left(\max _{i=1, m}\left|1-B_{i k}\right|+\max _{i=1, m} \int_{t_{k-1}-t_{k}}^{0} e^{-\lambda \theta} d \zeta_{i k}(\theta)\right) V\left(t_{k-1}+0\right)
\end{aligned}
$$

Combining the last estimate with (12) and (13), we derive

$$
V(t) \leq \prod_{k=1}^{i(0, t)}\left(\max _{i=\overline{1, m}}\left|1-B_{i k}\right|+\max _{i=\overline{1, m}} \int_{t_{k-1}-t_{k}}^{0} e^{-\lambda \theta} d \zeta_{i k}(\theta)\right) V(0), \quad t \geq 0 .
$$

Finally, by using the inequality (11) and the definitions of $V(t)$ and $y_{i}(t)$, we obtain the estimate (6) .

It is clear that inequality (6) guarantees global exponential stability of the equilibrium point $\mathbf{0}$ of the system without impulses (1). Further on, for three sets of additional assumptions on the impulse effects we will show that inequality (6) implies global exponential stability of the equilibrium point $\mathbf{0}$ of the impulsive system (11), (2).

Corollary 1. Let all conditions of Theorem 1 hold and

$$
\max _{i=\overline{1, m}}\left|1-B_{i k}\right|+\max _{i=\overline{1, m}} \int_{t_{k-1}-t_{k}}^{0} e^{-\lambda \theta} d \zeta_{i k}(\theta) \leq 1
$$

for all sufficiently large values of $k \in \mathbb{N}$. Then the equilibrium point $\mathbf{0}$ of the impulsive system (11), (2) is globally exponentially stable with Lyapunov exponent $\lambda$. 


\section{IMPROVED ESTIMATES VIA HARDY-POINCARÉ INEQUALITY}

In the previous corollary the global exponential stability was ensured by the rather small magnitudes of the impulse effects. Further we will show that we may have global exponential stability for quite large and even unbounded magnitudes of the impulse effects provided that these do not occur too often.

Corollary 2. Let all conditions of Theorem 1 hold and

$$
\limsup _{t \rightarrow \infty} \frac{i(0, t)}{t}=p<+\infty .
$$

Let there exist a positive constant $B$ satisfying the inequalities

$$
\max _{i=1, m}\left|1-B_{i k}\right|+\max _{i=\overline{1, m}} \int_{t_{k-1}-t_{k}}^{0} e^{-\lambda \theta} d \zeta_{i k}(\theta) \leq B
$$

and $p \ln B<\lambda$. Then for any $\tilde{\lambda} \in(0, \lambda-p \ln B)$ the equilibrium point $\mathbf{0}$ of the impulsive system (11), (2) is globally exponentially stable with Lyapunov exponent $\tilde{\lambda}$.

Similar conditions on the impulse effects were introduced in our previous paper [2].

Corollary 3. Let all conditions of Theorem 1 hold and there exist a constant $\kappa \in(0, \lambda)$ satisfying the inequality

$$
\max _{i=1, m}\left|1-B_{i k}\right|+\max _{i=1, m} \int_{t_{k-1}-t_{k}}^{0} e^{-\lambda \theta} d \zeta_{i k}(\theta) \leq e^{\kappa\left(t_{k}-t_{k-1}\right)}
$$

for all sufficiently large values of $k \in \mathbb{N}$. Then the equilibrium point $\mathbf{0}$ of the impulsive system (11), (2) is globally exponentially stable with Lyapunov exponent $\lambda-\kappa$.

A similar condition was introduced in the paper [17].

\section{Examples}

Denote $\varphi(t)=(|t+1|-|t-1|) / 2$. Let $\Omega$ be the unit ball in $\mathbb{R}^{3}$

$$
\Omega=\left\{x \in \mathbb{R}^{3}|| x \mid<1\right\}
$$

and let $\nabla^{2}$ denote the Laplacian in $\mathbb{R}^{3}$

$$
\nabla^{2} u_{i}=\frac{\partial^{2} u_{i}}{\partial x_{1}^{2}}+\frac{\partial^{2} u_{i}}{\partial x_{2}^{2}}+\frac{\partial^{2} u_{i}}{\partial x_{3}^{2}}, \quad i=1,2 .
$$




\section{HAYDAR AKÇA — VALÉRY COVACHEV — ZLATINKA COVACHEVA}

Consider the system

$$
\begin{aligned}
& \frac{\partial u_{1}(t, x)}{\partial t}=16 \nabla^{2} u_{1}(t, x)+\left(2+\sin u_{1}(t, x)\right) \\
& \times\left\{-2 u_{1}(t, x)+0.5 \arctan u_{1}(t, x)+0.3 \varphi\left(u_{2}(t, x)\right)+\right. \\
& +0.1 u_{1}\left(t-\frac{1}{2} \arctan t, x\right)+0.12 \arctan u_{2}\left(t-\frac{2}{3} \varphi(t), x\right)+ \\
& \left.+0.1 \int_{-\infty}^{0} u_{1}(t+\theta, x) d e^{\theta}+0.15 \int_{-\infty}^{0} u_{2}(t+\theta, x) d e^{\theta}\right\},
\end{aligned}
$$

$$
\begin{aligned}
& \frac{\partial u_{2}(t, x)}{\partial t}=20 \nabla^{2} u_{2}(t, x)+\left(3+\sin u_{2}(t, x)\right) \\
& \times\left\{-3 u_{2}(t, x)-0.6 \varphi\left(u_{1}(t, x)\right)+0.5 \arctan u_{2}(t, x)+\right. \\
& +0.16 u_{1}\left(t-1-\frac{1}{3} \varphi(t), x\right)-0.3 \arctan u_{2}\left(t-2-\frac{3}{4} \varphi(t), x\right)+ \\
& \left.+0.1 \int_{-\infty}^{0} u_{1}(t+\theta, x) d e^{\theta}-0.2 \int_{-\infty}^{0} u_{2}(t+\theta, x) d e^{\theta}\right\},
\end{aligned}
$$$$
\Delta u_{i}\left(t_{k}, x\right)=-B_{i k} u_{i}\left(t_{k}, x\right)+\int_{t_{k-1}-t_{k}}^{0} u_{i}\left(t_{k}+\theta, x\right) d \zeta_{i k}(\theta), \quad k \in \mathbb{N},
$$$$
\left.u_{i}\right|_{\partial \Omega}=0, \quad u_{i}(s, x)=\phi_{i}(s, x), \quad s \leq 0, \quad x \in \Omega, \quad i=1,2,
$$

where the initial data $\phi_{1}(s, x), \phi_{2}(s, x)$ satisfy

$$
\sup _{s \leq 0} \int_{\Omega}\left(\phi_{1}^{2}(s, x)+\phi_{2}^{2}(s, x)\right) d x<\infty .
$$

This system has a unique equilibrium point $(0,0)^{T}$ and assumptions A1-A7 hold with $n=3, \omega=1, R_{\Omega}=1$,

$$
\begin{gathered}
\underline{D}=\left(\begin{array}{cc}
16 & 0 \\
0 & 20
\end{array}\right), \quad \underline{\alpha}=\left(\begin{array}{cc}
1 & 0 \\
0 & 2
\end{array}\right), \quad \bar{\alpha}=\left(\begin{array}{cc}
3 & 0 \\
0 & 4
\end{array}\right), \quad \underline{\beta}=\left(\begin{array}{ll}
2 & 0 \\
0 & 3
\end{array}\right), \\
\tau_{11}=\pi / 4, \tau_{12}=2 / 3, \tau_{21}=4 / 3, \tau_{22}=11 / 4, \mu_{11}=1 / 2, \mu_{12}=2 / 3, \mu_{21}=1 / 3,
\end{gathered}
$$




$$
\begin{gathered}
\mu_{22}=3 / 4, K_{i j}(\lambda)=1 /(1-\lambda), i, j=1,2, \lambda_{0}=1, F=G=H=\left(\begin{array}{ll}
1 & 0 \\
0 & 1
\end{array}\right), \\
|A|=\left(\begin{array}{ll}
0.5 & 0.3 \\
0.6 & 0.5
\end{array}\right), \quad|B(\mu)|=\left(\begin{array}{ll}
0.2 & 0.36 \\
0.24 & 1.2
\end{array}\right), \quad|C|=\left(\begin{array}{ll}
0.1 & 0.15 \\
0.1 & 0.2
\end{array}\right),
\end{gathered}
$$

the matrix

$$
\mathcal{A}=\left(\begin{array}{cc}
96.528 & -2.43 \\
-3.76 & 119.06
\end{array}\right)
$$

is an $M$-matrix. Further on, the vector $\xi=(1,1)^{T}$ is such that $\xi^{T} \mathcal{A}=(92.368$, 116.63) has positive components. Let us denote by $\Phi_{j}(\lambda), j=1,2$, the left-hand sides of inequalities (5) for the given vector $\xi$. Then

$$
\begin{aligned}
& \Phi_{1}(\lambda)=\lambda+0.6 e^{\pi \lambda / 4}+0.96 e^{4 \lambda / 3}+\frac{0.7}{1-\lambda}-94.628 \\
& \Phi_{2}(\lambda)=\lambda+1.08 e^{2 \lambda / 3}+4.8 e^{11 \lambda / 4}+\frac{1.25}{1-\lambda}-123.76 .
\end{aligned}
$$

Since $\Phi_{1}(0.975279)=-60.52221507<0$ and $\Phi_{2}(0.975279)=-0.00143964<0$, we can take $\lambda=0.975279$. Theorem 1 is valid for the system (16).

If we use Hardy-Sobolev inequality, then inequalities (7) are satisfied with $\xi=(6,5)^{T}$ and $\lambda=0.02849$. Using just the nonpositivity of the reaction-diffusion operators cannot provide an estimate of the form (6).

Let us consider the impulsive conditions

$$
\begin{aligned}
& \Delta u_{1}\left(t_{k}, x\right)=-\frac{1}{2} u_{1}\left(t_{k}, x\right)+\frac{1}{4} \int_{-1}^{0} u_{1}\left(t_{k}+\theta, x\right) d e^{\theta}, \\
& \Delta u_{2}\left(t_{k}, x\right)=-\frac{1}{4} u_{2}\left(t_{k}, x\right)+\frac{1}{4} \int_{-1}^{0} u_{2}\left(t_{k}+\theta, x\right) d e^{\theta}, \quad t_{k}=k, k \in \mathbb{N} .
\end{aligned}
$$

Now

$$
\begin{aligned}
\max _{i=1,2}\left|1-B_{i k}\right|+\max _{i=1,2} \int_{t_{k-1}-t_{k}}^{0} e^{-\lambda \theta} d \zeta_{i k}(\theta) & 3 \\
& =\frac{3}{4}+\frac{1}{4} \int_{-1}^{0} e^{-\lambda \theta} d e^{\theta}=\frac{3}{4}+\frac{1-e^{\lambda-1}}{4(1-\lambda)}, \quad \lambda<1 .
\end{aligned}
$$

Obviously, inequalities (14) are valid for all $k \in \mathbb{N}$ and all $\lambda \in(0,1)$, in particular, for $\lambda=0.975279$. According to Corollary 1 the equilibrium point $(0,0)^{T}$ of system (16) with impulsive conditions (17) is globally exponentially stable with Lyapunov exponent 0.975279. 
Next consider the impulsive conditions

$$
\begin{aligned}
& \Delta u_{1}\left(t_{k}, x\right)=-100 u_{1}\left(t_{k}, x\right)+\int_{-10}^{0} u_{1}\left(t_{k}+\theta, x\right) d e^{\theta} \\
& \Delta u_{2}\left(t_{k}, x\right)=-50 u_{2}\left(t_{k}, x\right)+\int_{-10}^{0} u_{2}\left(t_{k}+\theta, x\right) d e^{\theta}, \quad t_{k}=10 k, \quad k \in \mathbb{N} .
\end{aligned}
$$

Now

$$
\begin{aligned}
\max _{i=1,2}\left|1-B_{i k}\right|+\max _{i=1,2} \int_{t_{k-1}-t_{k}}^{0} e^{-\lambda \theta} d \zeta_{i k}(\theta) & =99+\int_{-10}^{0} e^{-\lambda \theta} d e^{\theta} \\
& =99+\frac{1-e^{10(\lambda-1)}}{1-\lambda} \quad \text { for } \lambda \in(0,1)
\end{aligned}
$$

and we can take $B=107.8598086$ which is the value of the above expression for $\lambda=0.975279$. Further on, $p=0.1$, for $\lambda=0.975279$ we have $\lambda-p \ln B \approx$ $0.975279-0.1 \times 4.680832315 \approx 0.507196$. According to Corollary 2 , the equilibrium point $(0,0)^{T}$ of system (16) with impulsive conditions (18) is globally exponentially stable with Lyapunov exponent any $\lambda \in(0,0.507196)$, we can take $\lambda=0.5$.

Finally, let us consider the impulsive conditions

$$
\begin{aligned}
& \Delta u_{1}\left(t_{k}, x\right)=-(k+1) u_{1}\left(t_{k}, x\right)+k \int_{-2 k+1}^{0} u_{1}\left(t_{k}+\theta, x\right) d e^{\theta}, \\
& \Delta u_{2}\left(t_{k}, x\right)=-\left(k^{2}+1\right) u_{2}\left(t_{k}, x\right)+k^{2} \int_{-2 k+1}^{0} u_{2}\left(t_{k}+\theta, x\right) d e^{\theta}, \quad t_{k}=k^{2}, k \in \mathbb{N} .
\end{aligned}
$$

Now for $\lambda=0.975279$ inequality (15) becomes $42.45143805 k^{2} \leq e^{\kappa(2 k-1)}$. Obviously, for any $\kappa>0$ this inequality is valid for all natural $k^{\prime} s$ large enough. For instance, for $\kappa=0.1$ the inequality (15) holds for $k \geq 61$. Thus, according to Corollary [3, the equilibrium point $(0,0)^{T}$ of system (16) with the impulsive conditions (19) is globally exponentially stable with Lyapunov exponent equal to any $\lambda \in(0,0.975279)$.

The impulsive conditions similar to (17)-(19) were given in our previous paper [3]. 


\title{
IMPROVED ESTIMATES VIA HARDY-POINCARÉ INEQUALITY
}

\author{
REFERENCES
}

[1] ADIMURTHI: Hardy-Sobolev inequality in $H^{1}(\Omega)$ and its applications, Commun. Contemp. Math. 4 (2002), 409-434.

[2] AKÇA, H.-ALASSAR, R.-COVACHEV, V.-COVACHEVA, Z.-AL-ZAHRANI, E.: Continuous-time additive Hopfield-type neural networks with impulses, J. Math. Anal. Appl. 290 (2004), 436-451.

[3] AKÇA, H.-COVACHEV, V.: Impulsive Cohen-Grossberg neural networks with S-type distributed delays, Tatra Mt. Math. Publ. 48 (2011), 1-13.

[4] AKÇA, H.-ALASSAR, R.-COVACHEV, V.-COVACHEVA, Z.: Impulsive Cohen-Grossberg neural networks with S-type distributed delays and reaction-diffusion terms, Int. J. Math. Comput. 10 (2011), 1-12.

[5] BAO, S.: Global exponential robust stability of static reaction-diffusion neural networks with S-type distributed delays, in: 6th Internat. Symp. on Neural Networks (H. Wang et al., eds.), Wuhan, PRC, 2009, Adv. Intell. Soft Comput., Vol. 56, Springer-Verlag, Berlin, 2009, pp. 69-79.

[6] BERMAN, A.-PLEMMONS, R. J.: Nonnegative Matrices in Mathematical Sciences. Academic Press, New York, 1979.

[7] BREZIS, H.- VÁSQUEZ, J. L.: Blow-up solutions of some nonlinear elliptic problems, Rev. Mat. Univ. Complut. Madrid 10 (1997), 443-469.

[8] COHEN, M. A.-GROSSBERG, S.: Absolute stability of global pattern formation and parallel memory storage by competitive neural networks, IEEE Trans. Syst. Man Cybern. 13 (1983), 815-826.

[9] FIEDLER, M.: Special Matrices and Their Applications in Numerical Mathematics. Martinus Nijhoff, Dordrecht, 1986.

[10] GUAN, Z.-H.-CHEN, G.: On delayed impulsive Hopfield neural networks, Neural Netw. 12 (1999), 273-280.

[11] GUO, D. J.-SUN, J. X.-LIN, Z. I.: Functional Methods of Nonlinear Ordinary Differential Equations. Shandong Science Press, Jinan, 1995.

[12] HORN, R. A.-JOHNSON, C. R.: Topics in Matrix Analysis. Cambridge University Press, Cambridge, 1991.

[13] KAO, Y.-BAO, S.: Exponential stability of reaction-diffusion Cohen-Grossberg neural networks with S-type distributed delays, in: 6th Internat. Symp. on Neural Networks (H. Wang et al., eds.), Wuhan, PRC, 2009, Adv. Intell. Soft Comput., Vol. 56, Springer-Verlag, Berlin, 2009, pp. 59-68.

[14] LI, Z.-LI, K.: Stability analysis of impulsive Cohen-Grossberg neural networks with distributed delays and reaction-diffusion terms, Appl. Math. Modelling 33 (2009), 1337-1348.

[15] LI, K.-SONG, Q.: Exponential stability of impulsive Cohen-Grossberg neural networks with time-varying delays and reaction-diffusion terms, Neurocomputing 72 (2008), $231-240$.

[16] LIAO, X. X.-YANG, S. Z.-CHEN, S. J.-FU, Y. L.: Stability of general neural networks with reaction-diffusion, Sci. China Ser. F 44 (2001), 389-395.

[17] MOHAMAD, S.-GOPALSAMY, K.-AKÇA, H.: Exponential stability of artificial neural networks with distributed delays and large impulses, Nonlinear Anal. Real World Appl. 9 (2008), 872-888.

[18] PAN, J.-LIU, X.-ZHONG, S.: Stability criteria for impulsive reaction-diffusion Cohen-Grossberg neural networks with time-varying delays, Math. Comput. Modelling 51 (2010), 1037-1050. 


\title{
HAYDAR AKÇA — VALÉRY COVACHEV — ZLATINKA COVACHEVA
}

[19] SONG, Q.-CAO, J.: Exponential stability for impulsive BAM neural networks with timevarying delays and reaction-diffusion terms, Adv. Differ. Equ. 2007 (2007), 18 pp.

[20] SONG, Q.-CAO, J.: Stability analysis of Cohen-Grossberg neural network with both time-varying delays and continuously distributed delays, J. Comp. Appl. Math. 197 (2006), 188-203.

[21] WANG, M.-WANG, L.: Global asymptotic robust stability of static neural network models with S-type distributed delays, Math. Comput. Modelling 44 (2006), 218-222.

[22] ZHANG, Y.: Asymptotic stability of impulsive reaction-diffusion cellular neural networks with time-varying delays, J. Appl. Math. 2012 (2012), 17 pp.

[23] ZHANG, Y.-LUO, Q.: Global exponential stability of impulsive delayed reaction-diffusion neural networks via Hardy-Poincaré inequality, Neurocomputing 83 (2012), $198-204$.

Received July 2, 2012

\author{
Haydar Akça \\ Department of Applied Sciences and Mathematics \\ College of Arts and Sciences \\ Abu Dhabi University, P. O. Box 59911 \\ Abu Dhabi \\ $U A E$ \\ E-mail: hakca@uaeu.ac.ae \\ Valéry Covachev \\ Department of Mathematics $\&$ Statistics \\ College of Science, P. O. Box 36 \\ Sultan Qaboos University \\ Muscat 123 \\ SULTANATE OF OMAN \\ Institute of Mathematics \\ Bulgarian Academy of Sciences \\ Sofia \\ BULGARIA \\ E-mail: vcovachev@hotmail.com \\ valery@squ.edu.om \\ Zlatinka Covacheva \\ Middle East College \\ Muscat \\ SULTANATE OF OMAN \\ Higher College of Telecommunications and Post \\ Sofia \\ BULGARIA \\ E-mail: zkovacheva@hotmail.com
}

